\title{
Picosecond Spectroscopy of Solutions, Proteins and Photosynthetic Membranes
}

\author{
GRAHAM R. FLEMING, WILLIAM T. LOTSHAW, ROBERT J. \\ GULOTTY, MARY C. CHANG and JACOB W. PETRICH
}

Department of Chemistry and the James Franck Institute, The University of Chicago, 5735 South Ellis Avenue, Chicago, Illinois 60637, USA

The photophysics of tryptophan is discussed and a model capable of rationalizing the photophysics of almost twenty derivatives of tryptophan is proposed. The model is based on conformers about the $\mathrm{C}^{\alpha}-\mathrm{C}^{\beta}$ bond and the relative rates of charge transfer from indole to various electrophiles. Three new tryptophan derivatives were synthesized to test the model. Accurate predictions concerning the relative fluorescence lifetimes and the form of the fluorescence decay law are made for tryptophan and seventeen of its derivatives, including the three new derivatives.

The fluorescence decay kinetics from chloroplasts from green plants are described. New data are presented on the fluorescence decays obtained from genetic mutants of chlamydomonas which lack specific components of the photosynthetic apparatus. The interpretation of the green plant chloroplast data in terms of the two photosystems and the light harvesting array is discussed in terms of the data from the mutant algae. Our data indicate that the major short component $(\sim 100 \mathrm{ps})$ obtained in the fluorescence decay of chloroplasts results from photochemical trapping by both photosystem I and photosystem II.

A synchronously pumped dye laser which produces two, independently tunable, synchronized picosecond pulse trains will be described. An application of the laser to time and frequency resolved polarization spectroscopy will be given. The results are used to estimate the homogeneous linewidth of the $S_{0} \rightarrow S_{1}$ transition of a large molecule in solution. The optical heterodyne technique is applied to separate the real and imagninary contributions of the third order nonlinear susceptibility.

\section{INTRODUCTION}

This paper presents results of our studies in three areas: the photophysics of tryptophan and its simple derivatives; the mechanism of energy transfer in, and the layout of, the light harvesting array of green plants; and the ultrafast phase and energy relaxation processes 
associated with electronic transitions in solution. The experimental techniques are all based on cw mode locked dye lasers. In the first two topics the technique of time correlated single photon counting is used. We have described our apparatus in detail previously ${ }^{1,2}$ and will not dwell on it further except to note that recently we have improved the time resolution of our apparatus by about four-fold through the use of microchannel plate photomultipliers. Figure 1 shows a comparison of the same fluorescence decay measured with a conventional (a) and microchannel plate detector (b). The instrument function in Figure $1 \mathrm{~b}$ is 95 ps FWHM.

\section{PHOTOPHYSICS OF TRYPTOPHAN AND ITS DERIVATIVES}

\section{Introduction}

It is well-known that the fluorescence decay of tryptophan has great potential for use as a probe of the environments and motions of
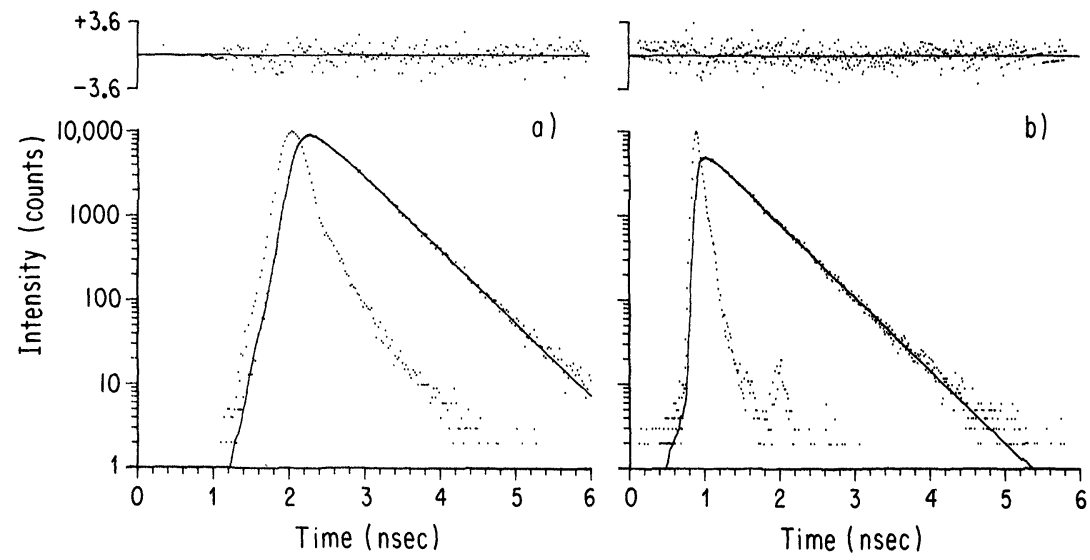

FIGURE 1 Comparison of conventional (a) and microchannel plate detection (b) in time correlated single photon counting. Both fluorescence decay curves are of oxazine in water at room temperature. The left curve was obtained with an Amperex PM31000. The right curve was obtained with an ITT 4169 microchannel plate photomultiplier. Similar results to (b) were obtained with a Hamamatsu $1645 U$ MCP. The instrument function full widths at half maximum for the PM31000 and the MCP are 320 ps and 95 ps respectively. The single exponential fits give fluorescence lifetimes of 483 ps and $494 \mathrm{ps}$ respectively; $\chi_{R}^{2}$ values are 0.815 and 0.918 respectively. 
proteins and smaller peptides and the fluorescence of tryptophyl residues has been widely exploited in this regard. ${ }^{3-5}$ Recently, however, it has been found that the fluorescence decay of isolated tryptophan is nonexponential but may be fit well to sums of two and three exponentially decaying components. ${ }^{6}$ Thus, the intrinsic nonexponentiality of tryptophan makes the protein fluorescence more difficult to interpret. In tryptophan, the nonexponential decay arises from two sources: one that is independent of $\mathrm{pH}$ from 4 to 8 and one that is strongly dependent on $\mathrm{pH}$ for $\mathrm{pH}>8$. We will not dwell on the $\mathrm{pH}$ dependent nonexponentiality, it is discussed in detail in Ref. 7. Rather, in this paper we will discuss the $\mathrm{pH}$ independent nonexponentiality which occurs for the $\mathrm{N}$-terminal tryptophyl compounds in the so-called $\mathrm{pH}$ independent range and for the C-terminal compounds (and others such as NATE) over the entire $\mathrm{pH}$ range. We present a model which is capable of rationalizing the photophysics of tryptophan and its simple analogs. The model accounts both for the magnitude of the fluorescence lifetimes and the presence or absence of nonexponential decay and makes several predictions which we have tested experimentally.

\section{The rotamer model}

Szabo and Rayner ${ }^{8}$ have used the rotamer model of Wahl and coworkers ${ }^{9,10}$ to explain the $\mathrm{pH}$ independent nonexponentiality. In their model different conformers of the indole ring are assumed to interconvert slowly compared with the excited state lifetime. If each conformer has a different lifetime a given compound will exhibit nonexponential decay. Such a model provides a rationale for fitting the observed decays to sums of exponentials, however it must be investigated and developed more thoroughly before it is accepted as an explanation for the observed tryptophyl photophysics. The points requiring scrutiny are: (1) the absorption and emission spectra of the rotamers, (2) extension of the model to compounds which exhibit nonexponential decay such as $\mathrm{N}$-acetyl tryptophan ethyl ester (NATE) and GlyTrp and to compounds which exhibit single exponential decay such as $\mathrm{N}$-acetyl tryptophamide (NATA) and indole 3-propronic acid, (3) the time scale of rotamer interconversion, (4) the possibility that a time dependent quenching process ${ }^{1}$ can explain the nonexponential decay, (5) the activation energies and frequency factors for the various 
derivatives and for the different decay components, and (6) the wide range of observed lifetimes- $-10.5 \mathrm{~ns}$ to $0.1 \mathrm{~ns}$.

\section{Charge transfer mechanism for nonradiative decay}

Flash photolysis studies of indole-containing species indicate that all such species exhibit intersystem crossing and photoionization as nonradiative pathways. ${ }^{11,12}$ These two processes alone are not able to explain the short lifetimes and low fluorescence quantum yields observed, for example, in tryptophan at $\mathrm{pH}$ 7. Many workers have thus considered the possibility of proton transfer from the protonated amino group ${ }^{1,13-15}$ to the indole ring and charge transfer from the indole ring to an acceptor ${ }^{16-18}$ as other modes of nonradiative decay. Two lines of reasoning have led us to conclude that charge transfer rather than proton transfer is responsible for the excited state quenching. Deuterated tryptophan and tryptophan have identical (nonexponential) decays in aprotic solvents such as DMSO. ${ }^{19}$ This result implies that the longer lifetimes observed in $\mathrm{D}_{2} \mathrm{O}$ are not due to intramolecular proton transfer but to a decrease of another nonradiative pathway. Second, the activation energies obtained from the low $\mathrm{pH}$ lifetime components of Trp and Trp-Gly are, within experimental error, the same as those obtained from the lifetime components of Gly-Trp. A more detailed discussion of these points is given in Ref. 7.

The conclusions presented below were arrived at by (1) combining the charge transfer process with the rotamer model, (2) using a model for charge transfer developed by Hopfield ${ }^{20}$ and (3) studying the time-resolved and steady state emission spectra of three model compounds which we have synthesized: 3-indolymethyl malonamide (AA), diethyl-3-indolylmethyl malonate (EE), and ethyl d-indolylmethyl malonate (AE). The structures of AA, EE and AE are shown in Figure 2, and their syntheses are described in Ref. 21.

\section{Time resolved fluorescence decay}

Table I summarizes the fluorescence decay data at $20^{\circ} \mathrm{C}$ for a range of tryptophyl derivatives including the three new compounds. If only a single decay time is reported the decay was found to be monoexponential. 
<smiles>NC(=O)C(Cc1c[nH]c2ccccc12)C(N)=O</smiles>

AA<smiles>CCOC(=O)C(Cc1c[nH]c2ccccc12)C(=O)OCC</smiles>

EE<smiles>CCOC(=O)C(Cc1c[nH]c2ccccc12)C(N)=O</smiles>

$A E$

FIGURE 2 Structures of the model compounds. AA: 3-indolylmethyl malonamide; EE: diethyl-3-indolylmethyl malonate; AE, ethyl-3-indolylmethyl malonate.

We have also carried out extensive studies of the temperature dependence of the nonradiative rate. ${ }^{7,21}$ Table II presents some of our data. Particularly striking in Table II is the finding that for the two components of the double exponential decays the activation energies are identical within experimental error and the differing lifetimes arise from changes in the frequency factor. Also notable is the similarity of the activation energies from derivative to derivative.

\section{The model for tryptophyl derivative photophysics}

We take as our initial assumption that the A, B and C rotamers (see Figure 3) do not interconvert quickly during the excited state lifetime. Second, we assume that in tryptophyl-containing compounds the dominant mode of nonradiative decay is charge transfer from the indole ring to an adjacent electrophile (e.g., a carbonyl carbon). Third, we suggest that this charge transfer, in addition to being a temperature dependent process, has a rate which is dependent on the proximity of the electrophile to the indole nitrogen and the electron affinity of the electrophile.

Given the above we suggest that the short decay component in NATE, for example, arises from rotamers B and C in which the ester 
TABLE I

Fluorescence lifetimes of tryptophyl compounds

\begin{tabular}{|c|c|c|c|}
\hline Compound & $A_{1}$ & $\tau_{1}(\mathrm{~ns})$ & $\tau_{2}(\mathrm{~ns})$ \\
\hline Indole 3-propionic acid ethyl ester ${ }^{8}$ & - & - & $3.36 \pm 0.04$ \\
\hline Tryptophan ethyl ester (pH 5) & $0.59 \pm 0.06$ & $0.26 \pm 0.02$ & $0.87 \pm 0.05$ \\
\hline Tryptophan ethyl ester (pH 9) & $0.27 \pm 0.05$ & $0.93 \pm 0.29$ & $2.40 \pm 0.22$ \\
\hline Indole 3-propionamide ${ }^{8}$ & - & - & $8.67 \pm 0.05$ \\
\hline Tryptophanamide ${ }^{\mathrm{a}}$ & $0.54 \pm 0.04$ & $1.14 \pm 0.01$ & $2.04 \pm 0.04$ \\
\hline Tryptophanamide (pH 9) & - & - & $7.01 \pm 0.02$ \\
\hline Indole 3-propionic acid ${ }^{8}$ & - & - & $10.76 \pm 0.08$ \\
\hline Tryptophan (pH 7) & $0.22 \pm 0.01$ & $0.62 \pm 0.05$ & $3.21 \pm 0.12$ \\
\hline Tryptophan (pH 11) ${ }^{1}$ & - & - & $8.18 \pm 0.24$ \\
\hline Trp-Gly & $0.23 \pm 0.01$ & $0.51 \pm 0.05$ & $1.91 \pm 0.06$ \\
\hline Trp-Gly $(\mathrm{pH} 11)^{7}$ & - & - & $7.90 \pm 0.24$ \\
\hline NATA & - & - & $2.95 \pm 0.03$ \\
\hline NATE & $0.32 \pm 0.03$ & $0.42 \pm 0.03$ & $1.70 \pm 0.03$ \\
\hline NAT & $0.33 \pm 0.08$ & $2.62 \pm 0.13$ & $5.18 \pm 0.10$ \\
\hline Gly-Trp & $0.46 \pm 0.03$ & $0.29 \pm 0.04$ & $1.26 \pm 0.01$ \\
\hline Gly-Trp-Gly & $0.60 \pm 0.03$ & $0.69 \pm 0.06$ & $1.69 \pm 0.09$ \\
\hline Gly-Trp-Gly-Gly & $0.30 \pm 0.03$ & $0.34 \pm 0.03$ & $1.23 \pm 0.01$ \\
\hline AA & - & - & $1.23 \pm 0.04$ \\
\hline $\mathrm{EE}$ & - & - & $0.14 \pm 0.01$ \\
\hline $\mathrm{AE}$ & $0.55 \pm 0.06$ & $0.11 \pm 0.01$ & $0.55 \pm 0.02$ \\
\hline
\end{tabular}

$\lambda_{\mathrm{ex}}=295 \mathrm{~nm}, \lambda_{\mathrm{em}} \geqslant 320 \mathrm{~nm}, T=20^{\circ} \mathrm{C}, \mathrm{pH}=5$ unless otherwise specified. The absence of a value for $A_{1}$ implies that the fluorescence decay was single exponential. $A_{1}+A_{2}=1$ for double exponential decays.

${ }_{p K_{a}} \sim 7$, ref. 8 .

$\mathrm{NAT}=\mathrm{N}$-acetyl tryptophan.

group is closer to the indole nitrogen than in rotomer A where the peptide bond is closer to the indole nitrogen. In NATA, however, where both groups adjacent to the indole ring have peptide-like carbonyls, A, B and C all have the carbonyl carbon equidistant from the indole nitrogen. Thus, according to our model, NATA would exhibit single exponential fluorescence, which it does. The study of the fluorescence decay of AA, EE and AE was carried out to test this explanation. These compounds are ideally suited for testing our hypothesis because in AA and EE the amide and ester groups are symmetrically placed about the indole ring-more so than in NATA. We expect, therefore, the fluorescence decay of AA and EE to be single exponential and to observe a shorter fluorescence lifetime in EE due to the higher electron affinity of the ester group. Furthermore, we would expect the fluorescence lifetime of $\mathrm{AE}$ to be double 
TABLE II

Summary of data from arrhenius plots

\begin{tabular}{lcc}
\hline \multicolumn{1}{c}{ Compound } & $E_{a}(\mathrm{kcal} / \mathrm{mole})$ & $A\left(\mathrm{~s}^{-1}\right)$ \\
\hline Trp $\left(\mathrm{pH} 11, \tau_{3}\right)^{1}$ & $12.3 \pm 0.4$ & $6.1 \pm 3.6 \times 10^{16}$ \\
Trp-Gly (pH 10.9, $\left.\tau_{3}\right)$ & $11.5 \pm 0.3$ & $1.3 \pm 0.7 \times 10^{16}$ \\
3-methyl indole & $12.6 \pm 0.3$ & $1.1 \pm 0.6 \times 10^{17}$ \\
Trp (pH 7, $\left.\tau_{1}\right)$ & $6.6 \pm 0.7$ & $1.2 \pm 1.3 \times 10^{14}$ \\
Trp (pH 7, $\left.\tau_{2}\right)$ & $6.7 \pm 0.2$ & $2.2 \pm 0.6 \times 10^{13}$ \\
Trp-Gly (pH 4.5, $\left.\tau_{1}\right)$ & $5.5 \pm 0.5$ & $2.2 \pm 1.9 \times 10^{13}$ \\
Trp-Gly (pH 4.5, $\left.\tau_{2}\right)$ & $5.4 \pm 0.1$ & $4.6 \pm 0.9 \times 10^{12}$ \\
Gly-Trp (pH 5.0, $\left.\tau_{1}\right)$ & $6.5 \pm 0.2$ & $2.2 \pm 0.8 \times 10^{14}$ \\
Gly-Trp (pH 5.0, $\left.\tau_{2}\right)$ & $5.5 \pm 0.1$ & $9.2 \pm 2.2 \times 10^{12}$ \\
NATE (pH 7.0, $\left.\tau_{1}\right)$ & $5.1 \pm 0.3$ & $1.3 \pm 0.6 \times 10^{13}$ \\
NATE (pH 7.0, & $4.9 \pm 0.1$ & $2.3 \pm 0.4 \times 10^{12}$ \\
NATA (pH 7.0) & $5.6 \pm 0.2$ & $3.9 \pm 0.9 \times 10^{12}$ \\
AE (pH 6.6, $\left.\tau_{1}\right)$ & $4.9 \pm 1.2$ & $3.8 \pm 8.3 \times 10^{13}$ \\
AE (pH 6.6, $\left.\tau_{2}\right)$ & $4.7 \pm 0.5$ & $5.6 \pm 4.5 \times 10^{12}$ \\
AA (pH 6.6) & $4.5 \pm 0.5$ & $1.5 \pm 1.3 \times 10^{12}$ \\
EE (pH 6.2) & $3.7 \pm 1.3$ & $4.2 \pm 9.1 \times 10^{12}$ \\
\hline
\end{tabular}

The activation energies and frequency factors correspond to the nonradiative rates associated with the various lifetime components which decrease in the order $\tau_{3}>\tau_{2}>\tau_{1}$.

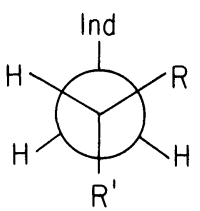

(A)<smiles>[R]C1C2CC3CC1C([3H])C(C2[R])C3[AlH2]</smiles>

(B)

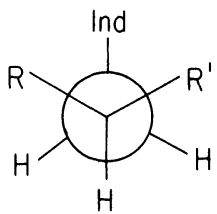

(C)

FIGURE 3 The $\boldsymbol{A}, \boldsymbol{B}$, and $\boldsymbol{C}$ rotamers about the $\mathrm{C}^{\alpha}-\mathrm{C}^{\beta}$ tryptophyl bond. Trp: $R=\mathrm{NH}_{3}^{+} \mathbf{R}^{\prime}=\mathrm{CO}_{2}^{-}$EE: $\mathrm{R}=\mathrm{COOEt} \mathbf{R}^{\prime}=\mathrm{COOEt}$. NATE: $\mathrm{R}=\mathrm{NHCOCH}_{3} \mathbf{R}^{\prime}=$ COOEt AE: $R=\mathrm{CONH}_{2} \mathbf{R}^{\prime}=$ COOEt. NATA: $R=\mathrm{NHCOCH}_{3} \mathbf{R}^{\prime}=\mathrm{CONH}_{2}$ AA: $\mathrm{R}=\mathrm{CONH}_{2} \mathbf{R}^{\prime}=\mathrm{CONH}_{2}$. 
exponential. Measurement of the fluorescence decays (Table I) completely confirmed these predictions. In AE our model suggests the short lifetime component is due to rotamers B and C; and the long lifetime component, to rotamer $\mathrm{A}$.

We now address the activation parameter data (Table II) in terms of our model. In the charge transfer model of Hopfield ${ }^{20}$ the rate of transfer from donor $(d)$ to acceptor $(a)$ is given by:

$$
k_{d a}=2 \pi / \hbar\left|T_{d a}\right|^{2} \int_{-\infty}^{\infty} D_{d}(E) D_{a}(E) d E
$$

where $T_{d a}$ is a matrix element containing information concerning the distance and orientation of the donor with respect to the acceptor. The $D_{d, a}(E)$ are the "electron removal" and "electron insertion" spectra of the donor and acceptor respectively. The product $D_{d}(E) D_{a}(E)$ is an exponential whose argument contains the ionization potential of the donor and the electron affinity of the acceptor and is thus responsible for the observed activation energy. Thus, to take a specific example, in the context of Hopfield's model, the decrease in the fluorescence lifetime components of Gly-Trp with respect to those of Trp-Gly and the constancy of the $E_{a}$ 's derived from these lifetimes can be attributed solely to the preferred quenching orientation of the peptide carbonyl in Gly-Trp as was initially suggested by Werner and Forster. ${ }^{17}$

It is generally accepted that esters are more electrophilic than amides or peptide bonds which in turn are more electrophilic than carboxylate groups. Hence we expect the $D_{d}(E) D_{a}(E)$ term to give rise to different $E_{a}$ 's for zwitterionic Trp and for NATE. Table II shows this trend is present.

As mentioned in the introduction, any satisfactory description of the tryptophyl photophysics must not only be able to explain the presence (or absence) of nonexponential fluorescence decay, but must also predict the relative fluorescence lifetimes of the tryptophyl compounds. As the indole is the donor in all cases we need only consider the relative electron affinities of the various acceptors. A suitable parameter (in the absence of literature values of electron affinities) is the acidity of the hydrogens $\alpha$ to the carbonyl carbon. ${ }^{22} \mathrm{~A}$ good correlation between acidity and ability to quench indole fluorescence is obtained. ${ }^{21}$ 


\section{Summary}

Based on the discussion presented above we can make the following statements concerning the nonexponentiality of the tryptophancontaining compounds in the light of our model.

1) The presence of two groups of different electron affinity attached to the $\alpha$-tryptophyl carbon will give rise to double exponential decay. This can be seen in NATE, NAT, and AE as compared with NATA, $\mathrm{AA}$, and EE.

This phenomenon is also observed in the high pH species of tryptophan and tryptophanamide as compared with the low $\mathrm{pH}$ species of tryptophan and tryptophanamide, and of tryptophan ethyl ester at all $\mathrm{pH}$ 's. For example, at $\mathrm{pH} 7$ the fluorescence lifetime of tryptamine $\left(p K_{a}=10.2^{23}\right)$ is $\sim 6 \mathrm{~ns}$; but when the $\mathrm{pH}$ is raised to 11 , a $9 \mathrm{~ns}$ component appears. This is near the lifetime of indole 3-propionic acid: $\sim 10.5 \mathrm{~ns}$. Thus at high $\mathrm{pH},-\mathrm{CO}_{2}^{-}$and $-\mathrm{NH}_{2}$ are equally poor electrophiles and anionic tryptophan exhibits single exponential decay. A similar argument may be made to tryptophanamide. Tryptophan ethyl ester, however, is different in that at all pH's the electrophilicity of the amino group is lower than that of the ester group. Thus, tryptophan ethyl ester is double exponential over the entire $\mathrm{pH}$ range.

2) Tryptophyl compounds which have only one substituent attached to the $\alpha$ carbon are single exponential because the conformers obtained by rotation about the $\mathrm{C}^{\alpha}-\mathrm{C}^{\beta}$ bond also possess values of $\phi, \psi$ and the angle formed by rotation about $\mathrm{C}^{\beta}-\mathrm{C}^{\gamma}$ which maintain equivalent quencher-indole nitrogen distances. Similar considerations must be made for tryptophyl compounds with two carbonyl groups attached to the $\alpha$ carbon. Examination of space-filling models for NATA or NATE, for example, shows that it is rotation about these bonds which prevents both carbonyl carbons from being equidistant from the indole nitrogen in conformer $\mathrm{C}$. Such rotation prevents the lifetime of the NATA C conformer from being shorter than that of the $\mathrm{A}$ and $\mathrm{B}$ conformers.

\section{Conclusion}

The qualitative features of the fluorescence decay of tryptophan and seventeen of its derivatives are rationalized by our simple model. Our 
model makes definite predictions, is easily tested, and seems consistent with a large number of experimental observations. In particular, the form of the decay law is accurately predicted in all cases; and predictions of relative lifetimes based on the charge transfer rate for different conformers about the $\mathrm{C}^{\alpha}-\mathrm{C}^{\beta}$ bond are quite good.

\section{ENERGY TRANSFER IN PHOTOSYNTHETIC LIGHT HARVESTING ARRAYS}

\section{Introduction}

The photosynthetic light harvesting array of higher plants and algae consists of three major collections of chlorophyll (chl) molecules. They are antenna chlorophyll associated with the two reaction centers, PSI and PSII, and the chl $a / b$ protein. The nature of excitation energy transfer in and among these units is, at present, poorly understood. We and others have examined the fluorescence decay kinetics of chloroplasts and find ${ }^{24,25}$ that the decays are largely bi-exponential although a weak longer component is also present. In this paper we attempt to explain the origin of these components by comparison of the fluorescence decay properties of Chlamydomonas mutants lacking various parts of the light harvesting array, i.e., PSI, PSII, chl $a / b$ protein, with the fluorescence decay properties of the wild-type alga.

\section{Experimental}

A. Fluorescence decay measurements Fluorescence decays were recorded by the time correlated single photon counting technique using an Amperex PM31000A photomultiplier. Samples were flowed to avoid preillumination, and laser pulse intensities in the range $10^{8}-10^{12}$ photons $\mathrm{cm}^{-2}$ pulse $\mathrm{s}^{-1}$ gave identical decays. The laser repetition rate was reduced from $91 \mathrm{MHz}$ to $45 \mathrm{kHz}$ using two electrooptic modulators in series. Contrast ratios of 200:1 to 1000:1 for selected versus rejected pulse areas were achieved depending on Pockell cell alignment and excitation wavelength.

B. Biochemical characterization of wild-type and mutant chlamydomonas strains The Chlamydomonas reinhardii wild-type and mutant cells were grown heterotrophically according to Spreitzer 
and Mets. ${ }^{26}$ The amounts of $\operatorname{chl} a$ and $\operatorname{chl} b$ in the Chlamydomonas cells were routinely determined spectroscopically using the Arnon equations ${ }^{27}$ for absorption spectra of the cell chlorophyll extracted into $80 \%$ acetone. As this method is not very accurate for samples with high $\operatorname{chl} a$ to $\operatorname{chl} b$ ratios we checked our results using the fluorescence method of Boardman et al. ${ }^{28}$ and by HPLC analysis. ${ }^{29}$ The chlorophyll to $\mathrm{P} 700$ ratios were measured by differential absorption spectroscopy. ${ }^{30}$ Flashing light $\mathrm{O}_{2}$ evolution ${ }^{31}$ of intact cells was performed using two Stroboslaves strobes yielding synchronous $5 \mu \mathrm{s}$ flashes at $10 \mathrm{~Hz}$ and a Clark-type oxygen electrode. ${ }^{32}$

\section{Results}

A. Biochemical characteristics of wild-type vs. mutant chlamydomonas A comparison of the chlorophyll and reaction center composition of wild-type (WT2137) versus $\operatorname{chl} b$ deficient (DS521) Chlamydomonas is given in Table III. The DS521 strain is deficient in chlorophyll $b$, and has $\sim 56 \%$ less chlorophyll per reaction center compared with the WT2137 strain. Sodium dodecyl sulfate (SDS) and lithium dodecyl sulfate (LDS) native and non-native electrophoretic gels ${ }^{33,34}$ show that the DS521 strain is largely deficient in $\operatorname{chl} a / b$ protein. The respective $\operatorname{chl} a: \operatorname{chl} b$ ratios are consistent with loss of $\operatorname{chl} a / b$ protein only.

The photosystem I mutant (12-7) shows no detectable P700 signal and no CPI band on SDS native and non-native electrophoretic gels. ${ }^{33}$ The photosystem II mutant (8-36) is missing polypeptides associated with PSII $^{35}$ and shows no DPC-DPIP reduction activity. ${ }^{36}$ The PSI and PSII mutants both contain approximately wild-type levels of chlorophyll $a / b$ protein taking into account the loss of one of the two photosystems and associated core chlorophyll (chl $a: \operatorname{chl} b \sim 2.0$ ).

TABLE III

Chlorophyll and reaction center composition of wild-type and DS mutant chlamydomonas

\begin{tabular}{lccc}
\hline Sample & $\mathrm{Chl} a / \mathrm{chl} b$ & $\mathrm{Chl} / \mathrm{P700}$ & $\mathrm{Chl} / \mathrm{O}_{2}$ \\
\hline Wild-type & 2.6 & 320 & 2500 \\
DS mutant & 8.0 & 140 & 1100 \\
\hline
\end{tabular}


B. Fluorescence decay properties Wild-type Chlamydomonas cells exhibit fluorescence decay kinetics which are nonexponential. The decays are best fit to a sum of three exponential components where two of these components represent the decay of $99 \%$ of the absorbed excitation. Table IV shows that the wild-type (WT2137) decay parameters are very similar to those measured for barley and spinach chloroplasts. $^{24}$ The decay properties of the DS521 mutant are similarly nonexponential, however there is a marked decrease in the major lifetime component, $\tau_{1}=44 \mathrm{ps,}$, whereas $\tau_{1}=100 \mathrm{ps}$ in the WT2137 mutant. The PSII and PSI mutants both have a short lifetime component characteristic of photochemical trapping. In addition, they show an increase in the lifetime of $\tau_{2}$ and pre-exponential factor $\boldsymbol{A}_{3}$ relative to the wild-type.

\section{Discussion}

The biochemical data indicate that the DS521 mutant contains $\sim 90 \%$ less $\operatorname{chl} a / b$ protein than the WT2137 Chlamydomonas. The chlorophyll to P700 ratio and chlorophyll per evolved $\mathrm{O}_{2}$ measurements show that the DS521 mutant has 56\% less chlorophyll per PSI or PSII reaction center relative to the wild-type. Hence, we can infer that the relative number and activity of PSI and PSII are nearly constant in both strains, the only change being the loss of $\operatorname{chl} a / b$ protein. Since the major change in the decay characteristics of the

TABLE IV

Summary of fluorescence decay data chloroplasts. The data were fit to

\begin{tabular}{|c|c|c|c|c|c|c|}
\hline Sample & $\tau_{1}(\mathrm{ps})$ & $\tau_{2}(\mathrm{ps})$ & $\tau_{3}(\mathrm{ps})$ & $A_{1}$ & $A_{2}$ & $A_{3}$ \\
\hline Dark-adapted Barley & 112 & 380 & 2214 & 79 & 21 & 0.7 \\
\hline Unflowed Barley & 134 & 675 & 2123 & 68.8 & 25.4 & 5.9 \\
\hline Dark-adapted Spinach & 87 & 318 & 2312 & 62 & 37 & 0.7 \\
\hline Chlamydomanas WT & 100 & 462 & 2871 & 73 & 26 & 0.8 \\
\hline $\begin{array}{l}\text { Chlamydomonas Chl } b \\
\text { deficient DS521 }\end{array}$ & 44 & 408 & 2676 & 92 & 7 & 0.6 \\
\hline $\begin{array}{l}\text { Chlamydomonas PSII } \\
\text { mutant 8-36 (lacks PSII) }\end{array}$ & 67 & 772 & 1973 & 71 & 18 & 11 \\
\hline $\begin{array}{l}\text { Chlamydomonas PSI } \\
\text { mutant 12-7 (lacks PSI) }\end{array}$ & 266 & 900 & 3177 & 47 & 45 & 7 \\
\hline
\end{tabular}


DS521 relative to WT2137 was the decrease in the short lifetime component from $100 \mathrm{ps}$ to $44 \mathrm{ps}$, this implies that the $100 \mathrm{ps}$ lifetime component represents photochemical trapping of excitation originating from chl $a / b$ protein as well as the core chlorophyll about each reaction center.

In a recent paper ${ }^{24}$ we suggested two plausible phenomenological models for the origin of the nonexponential decay kinetics of chloroplasts. The DS521 versus WT2137 comparison supports the second model where the major short ( $100 \mathrm{ps)} \mathrm{component} \mathrm{represents} \mathrm{transfer}$ of excitiation from anywhere in the chlorophyll antenna array to either PSI or efficiently quenching PSII. The intermediate lifetime component represents energy transfer to PSII in a less efficiently quenching redox state.

Our preliminary measurement of the PSI and PSII mutants show that both have short lifetimes $\left(\tau_{1}=266,67 \mathrm{ps}\right.$ respectively, see Table IV) which can be associated with primary photochemistry. The longer $\tau_{1}$ lifetime of the PSI mutant may represent an average of transfer of energy to PSII centers of different quenching efficiency and the absence of a 100 ps component in this decay deserves further scrutiny. The increases of $\tau_{2}$ and $A_{3}$ may be due to an increase of chl $a / b$ protein decoupled from quenching reaction centers. Lotshaw $e t a l^{2}$ obtained similar lifetimes and weights for $\tau_{2}, \tau_{3}$ (Table IV) for aggregated chl $a / b$ proteins in phosphotadylcholine vesicles. We also find similar behavior in preilluminated chloroplasts, where PSII centers are closed. We are currently preparing double mutant cultures in order to test and refine these ideas.

\section{TIME AND FREQUENCY RESOLVED POLARIZATION SPECTROSCOPY}

\section{Introduction}

Time resolved polarization spectroscopy (referred to in our previous publications ${ }^{37,38}$ as anisotropic absorption) is a powerful and sensitive technique for the study of dynamics in the liquid phase. On the subpicosecond time scale or in combination with frequency resolution, information can be obtained on the ultrafast dephasing and energy relaxation processes associated with electronic transitions. On the picosecond and longer time scale the experiments provide detailed 
information on transitions between electronic states and on the motion of molecules in these electronic states. We have recently presented a general description of these latter type of experiments ${ }^{39}$ and will not consider them in detail here. Instead we will describe some recent studies in which we have investigated the spectrum of the third order susceptibility and utilized the optical heterodyne technique ${ }^{40,41}$ to detect, separately, the real and imaginary contributions to the susceptibility. Our experiments are based on a double color synchronously pumped dye laser which is described in detail in the next section.

\section{Experimental}

We have constructed a version of a tandem, intersecting cavity dye laser first described by Frigo et al. $^{42}$ This dye laser, shown schematically in Figure 4, employs the conventional cavity of a Rhodamine 6-G synchronously pumped dye laser to which a second dye jet, with focusing and recollimating mirrors, has been added. The absorption band of the second dye overlaps the lasing spectrum of the first, and acts as a saturable absorber in the first cavity. This permits the first cavity to be operated near threshold (i.e., with good mode locking) when the output coupling is low $(\sim 2-5 \% \mathrm{~T})$. The second cavity is then defined by placing an additional set of mirrors around the second dye jet and at either end of the cavity. Care must be taken to position the high reflecting end mirror of the second cavity at a distance from the second dye jet that is slightly greater than the distance in the first

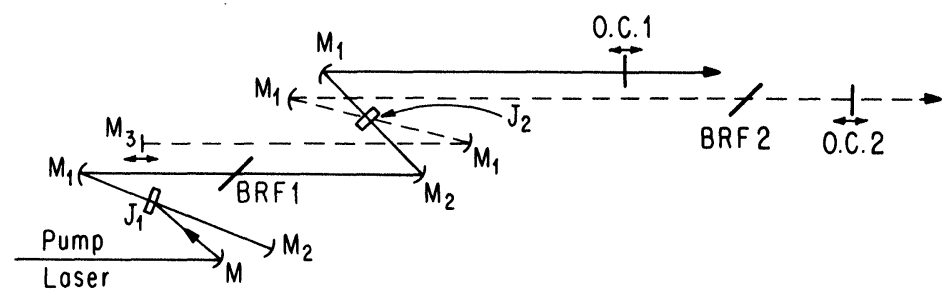

FIGURE 4. Optical arrangement for the intersecting cavity dye laser. The dashed line represents the cresyl violet laser and the solid line the rhodamine 6-G laser. Mirrors $M_{1}$ are $7.5 \mathrm{~cm}$ radius, $M_{2}$ is $5 \mathrm{~cm}$ and $M_{3}$ is flat. The distance along the solid line from $J_{2}$ (the cresyl violet jet) to $\mathrm{OCl}$ is the distance $\mathrm{D}_{1}$ defined in the text. Likewise, the distance along the dashed line from $M_{3}$ to $J_{2}$ is $D_{2}$. 
cavity from the saturable absorber to the output coupler of the first cavity $\left(D_{1} \cong D_{2} \pm \Delta d\right.$ in Figure 4). The distance $\Delta d$ is defined by the time interval over which the integral of the $\sim 5 \mathrm{ps}$ "pumping" pulse of the first dye laser rises from 10 to $90 \%$ of its maximum value. This integral defines the temporal profile of the gain in the second dye laser. Only when the dye laser pulses in the second cavity overlap this region of the gain profile will the synchronization between the pulses in each cavity be reinforced. This distance corresponds to a translation of the second cavity high reflector of $\sim 2 \mathrm{~mm}$. This configuration results in two independently tunable dye lasers which intersect at the gain medium of the second cavity. The design utilizes the high intracavity power of the ion laser pumped dye laser to drive the gain medium of the second cavity with the mode locking advantage of very short pumping pulses. We have constructed the dye laser from two Coherent CR599 dye laser modules, one of which was modified to accomodate the extra mirror set around the dye jet shown in Figure 5. The mirrors about the second dye jet are $7.5 \mathrm{~cm}$ radius, $\frac{11}{4}$ diameter, and were chosen primarily for reasons of convenience. A third flat mirror diverts the laser beam in the second cavity through a 2 plate birefringent filter to $\mathrm{a} \sim 2 \% \mathrm{~T}$ flat output mirror.

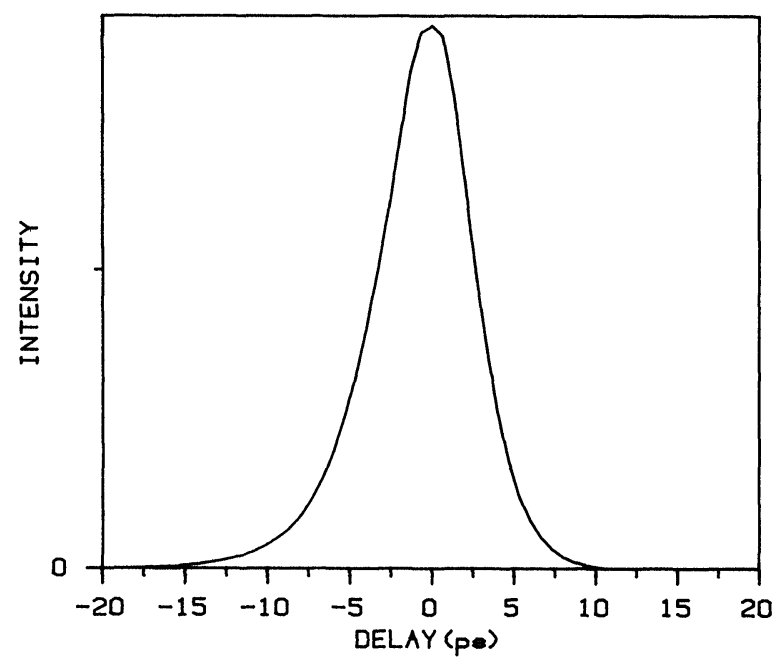

FIGURE 5 Cross correlation of the two output pulse trains from the double color laser. The trace has a FWHM of 6 ps. 
In this configuration, with the first dye jet pumped by the $5145 \AA$ line $(1.3 \mathrm{~W})$ of a mode locked argon laser, the average power outputs of the Rhodamine 6-G and Cresyl Violet dye lasers are $\sim 120$ and $\sim 12 \mathrm{~mW}$. The ouput pulses are temporally well correlated, the measured cross correlation function having a $\sim 6 \mathrm{ps}$ full width at half maximum, see Figure 5. The parameters most crucial to the attainment of a minimum cross correlation are (i) finding the optimal output coupling for each cavity, (ii) a well mode locked ion laser, (iii) precise matching of the second cavity gain medium to end mirror distance to the first cavity saturable absorber to output mirror distance (note that second cavity gain medium is equivalent to first cavity saturable absorber). We note that while the available average power in our laser is slightly less than that reported by Frigo et al. the timing "jitter" between the outputs of each dye laser is substantially less, and close to the theoretical minimum for two pulses of widths (from autocorrelations) $\sim 5 \mathrm{ps}$ (R6-G) and 3-4 ps (Cresyl Violet). The frequency bandwidths of the dye lasers are $140 \mathrm{GHz}$ for the Rhodamine laser and $\sim 120 \mathrm{GHz}$ for the Cresyl Violet laser. The Cresyl Violet laser has a substantial tuning range at Rhodamine 6-G lasing wavelengths between 5800 and $6000 \AA$ (the maximum of $C V$ absorption). It can be tuned between $6250 \AA$ and $6600 \AA$ with average powers $>5 \mathrm{~mW}$ without disturbing the cross correlation width of $\sim 6 \mathrm{ps}$ (a simple adjustment of the cavity length is necessary). Since the "pump" pulse duration in the second cavity is short $(\sim 5 \mathrm{ps})$, maximum average power and minimum cross correlation width are achieved at the same cavity length. The combination of this double color laser with our high repetition rate amplifier system ${ }^{43}$ will provide a very versatile spectroscopic source.

\section{Polarization spectroscopy}

Our preliminary studies were carried out on the dye molecule oxazine (Oxazine-725 Exciton) dissolved in methanol at $\sim 10^{-4} \mathrm{M}$. One reason for interest in oxazine is that our earlier studies showed that for excitation and probing at $590 \mathrm{~nm}$ the polarization spectroscopy signal was almost entirely due to birefringence. ${ }^{37}$ In the present experiments excitation was at $595 \mathrm{~nm}$ (fixed) and the probe wavelength was scanned from 625-655 nm. As Figure 6 shows, the excitation wavelength is in the short wavelength "hump" of the absorption 


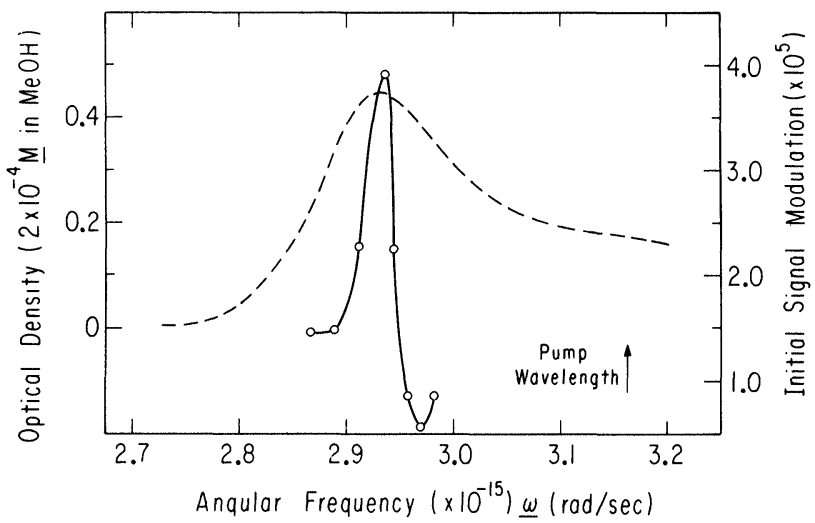

FIGURE 6 Optical absorption spectrum (dashed curve) and initial height of polarization spectroscopy signal as a function of probe wavelength (solid curve). The pump wavelength is arrowed. In this experiment the probe is linearly polarized at $45^{\circ}$ to the pump polarization.

spectrum (arrow) and the probe in the major long wavelength band. The solid curve in Figure 6 is the normalized initial signal height plotted as a function of probe wavelength. This curve corresponds to the spectrum of $\left|\chi^{(3)}\right|^{2}$. There is no coherent coupling between such dissimilar pump and probe wavelengths. By attempting to remove the signal by (a) rotating the analyzer or (b) inserting a waveplate between sample and analyzer the signal was found to be roughly $70 \%$ dichroic and 30\% birefringent. The solid curve in Figure 6 indeed resembles a roughly $2: 1$ superposition of a Lorenzian and a dispersion line.

\section{Optical heterodyne spectroscopy}

The birefringent and dichroic contributions to the signal in Figure 6 arise from the real and imaginary parts of the third order susceptibility:

$$
\chi^{(3)}=\chi^{(3) \prime}+i \chi^{(3) \prime \prime}
$$

By adapting the methods of optical heterodyne spectroscopy ${ }^{40}$ used in coherent Raman spectroscopy it is possible to measure the contributions of the real and imaginary parts of $\chi^{(3)}$ separately. As described in Eesley et al. $^{40}$ and by Owyoung ${ }^{41}$ and confirmed by our Jones 
matrix analysis ${ }^{37}$ modification of the polarization of the probe can be used to (a) selectively enhance one component of the signal and (b) linearize the response. Thus, we are now in a position to measure $\operatorname{Im} \chi^{(3)}$ or $\operatorname{Re} \chi^{(3)}$ directly. We also note that the heterodyne technique is useful for the longer time studies of orientational motion and level kinetics since the decay is now in general a sum of exponentials rather than the square of the sum. Thus the cross terms are eliminated and, for example, nonexponential rotational correlation functions should become detectable. For the present case, Eq. (1) shows the origin of the heterodyne effect. The detected signal is proportional to

$$
I(t)=\frac{n c}{8 \pi}\left|E_{0}(t)+E_{s}(t)\right|^{2}=I_{0}(t)+I_{s}(t)+\frac{n c}{4 \pi} \operatorname{Re}\left[E_{0}^{*}(t) \cdot E_{s}(t)\right]
$$

where $I_{s}(t)$ in the signal arises from the sample and $I_{0}(t)$ is the signal arising from the change in probe polarization from that giving complete extinction. If $E_{0} \gg E_{s}$ then the heterodyne term $n_{s} c / 4 \pi \operatorname{Re}\left[E_{0}^{*}(t) \cdot E_{s}(t)\right]$ makes the dominant contribution to the signal if the pump beam is chopped and the lockin amplifier rejects the d.c. signal $I_{0}(t)$. If the probe beam is linearly polarized and tipped toward the pump polarization the heterodyne term contains only the dichroic contribution. If the probe remains at $+45^{\circ}$ but is made slightly elliptically polarized the heterodyne term contains only the birefringent contribution.

Experimentally we have observed the linearization and increase in magnitude of the heterodyne signal for the dichroic case. The decay time for the signal exactly doubles as expected for single exponential decay (Figure 7). Recording of the heterodyne spectrum of $\operatorname{Im} \chi^{(3)}$ is complicated by fluorescence interference resulting from chopping the strong pump beam rather than the weak probe. We are currently implementing a double modulation scheme that will remove this difficulty. Our present preliminary results confirm that the dichroic heterodyne signal consists of a single Lorentzian like peak.

\section{Estimate of dephasing time}

In the two level plus reservoir model introduced by Song et al. ${ }^{44}$ the FWHM of $\operatorname{Im} \chi^{(3)}$ is $4 \Gamma_{2}$ where $\Gamma_{2}^{-1}=T_{2}$, the dephasing time. Although we clearly have at least three levels involved in our case we will use this expression for the purpose of estimating $T_{2}$ in order to compare 


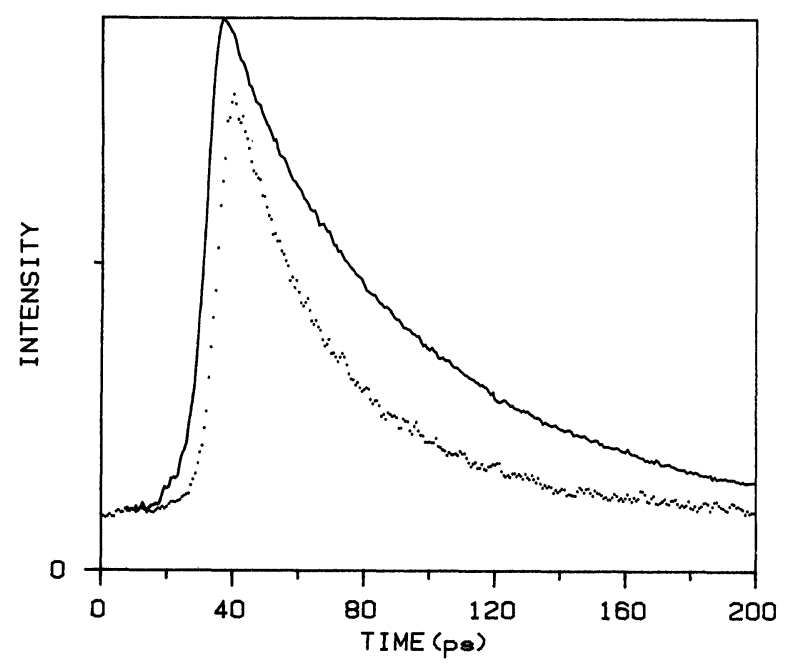

FIGURE 7. Comparison of normal $(\ldots \ldots)$ and heterodyne $(\longrightarrow)$ trace. The decays are for oxazine in methanol and at $30 \mathrm{ps}$ and $60 \mathrm{ps}$ for the normal and heterodyne curves respectively.

with the $T_{2}$ value obtained by assuming the absorption band is homogeneous. If we assume that the absorption band of oxazine is composed of two overlapping homogeneous bands (Figure 6) we estimate $T_{2} \sim 15-20 \mathrm{fs}$. The polarization spectroscopy signal contains both dichroic and birefringent contributions and is not strictly suitable for evaluating $T_{2}$. However, from Figure 6 we estimate that $4 \Gamma_{2}$ lies in the range $4 \times 10^{13}-2.5 \times 10^{13} \mathrm{~s}^{-1}$ (angular frequency). Thus we find that $T_{2}$ lies in the range 100-200 fs and that the absorption exhibits considerable inhomogeneous broadening. A considerable range of dephasing times has been reported for dye molecules in solution ${ }^{44-46}$ from 20-200 fs. We believe our technique to be less ambiguous than the Rayleigh mixing method of Yajima ${ }^{45,46}$ and plan to study the influence of solvent, solute and temperature on our lineshapes.

\section{Acknowledgement}

This work was supported by grants from NIH, NSF and the Petroleum Research Fund of the ACS. The photosynthetic studies were carried out in collaboration with R. S. Alberte and L. Mets of the Biology Department, The University of Chicago. 


\section{References}

1. R. J. Robbins, G. R. Fleming, G. S. Beddard, G. W. Robinson, P. J. Thistlethwaite and G. J. Woolfe, J. Am. Chem. Soc. 102, 6271 (1980).

2. W. T. Lotshaw, G. R. Fleming and R. S. Alberte, Biochim. Biophys. Acta 682, 75 (1982).

3. I. Munro, I. Pecht and L. Stryer, Proc. Natl. Acad. Sci. U.S.A. 76, 56 (1979).

4. M. R. Eftink and C. A. Ghiron, Biochemistry 15, 672 (1976).

5. J. B. A. Ross, K. W. Rousslang and L. Brand, Biochemistry 20, 4361 (1981).

6. E. Gudgin, R. Lopez-Delgado and W. R. Ware, Can. J. Chem. 59, 1037 (1981).

7. M. C. Chang, J. W. Petrich, D. B. McDonald and G. R. Fleming, J. Am. Chem. Soc. in press (1983).

8. A. G. Szabo and D. M. Rayner, J. Am. Chem. Soc. 102, 554 (1980).

9. B. Donzel, P. Gauduchon and Ph. Wahl, J. Am. Chem. Soc. 96, 801 (1974).

10. P. Gauduchon and Ph. Wahl, Biophys. Chem. 8, 87 (1978).

11. D. V. Bent and E. Hayon, J. Am. Chem. Soc. 97, 2612 (1975).

12. J. C. Mialocq, E. Amouyal, A. Bernas and D. Grand, J. Phys. Chem. 86, 3173 (1982).

13. R. W. Ricci, Photochem. Photobiol. 12, 67 (1970).

14. S. S. Leher, J. Am. Chem. Soc. 92,3459 (1970).

15. I. Weinryb and R. F. Steiner, Biochemistry 7, 2488 (1968).

16. R. W. Ricci and J. M. Nesta, J. Phys. Chem. 80, 874 (1976).

17. T. C. Werner and L. S. Forster, Photochem. Photobiol. 29, 905 (1979).

18. G. R. Fleming, J. M. Morris, R. J. Robbins, G. J. Woolfe, P. J. Thistlethwaite and G. W. Robinson, Proc. Natl. Acad. Sci. U.S.A. 75, 4652 (1978).

19. E. Gudgin, R. Lopez-Delgado and W. R. Ware, private communication.

20. J. J. Hopfield, Proc. Natl. Acad. Sci. U.S.A. 71, 3640 (1974).

21. J. W. Petrich, M. C. Chang, D. B. McDonald and G. R. Fleming, J. Am. Chem. Soc. in press (1983).

22. D. J. Cram, Fundamentals of Carbanion Chemistry (Academic Press, Inc., New York, 1965), 1st ed., Chapter 1, pp. 1-84.

23. J. W. Bridges and R. T. Williams, Biochem. J. 107, 225 (1968).

24. R. J. Gulotty, R. S. Alberte and G. R. Fleming, Biochem. Biophys. Acta 682, 322 (1982).

25. W. Haehnel, J. A. Nairn, P. Reisberg and K. Sauer, Biochim. Biophys. Acta. 680, 161 (1982).

26. R. J. Spreitzer and L. Mets, Plant. Physiol. 67, 565 (1981).

27. D. I. Arnon, Plant. Physiol. 24, 1 (1933).

28. N. K. Boardman and S. W. Thorne, Biochim. Biophys. Acta. 253, 222 (1971).

29. A. Friedman, unpublished results.

30. W. E. Dietrich and J. P. Thornber, Biochem. Biophys. Acta. 245, 482 (1971).

31. R. Emerson and W. Arnold, J. Gen. Physio. 15, 391 (1932).

32. J. M. Clark, Experimental Biochemistry (W. H. Freeman, San Francisco, 1964), 2nd ed.

33. U. K. Laemmli, Nature (London) 227, 6801 (1970).

34. P. Delepelaire and H.-N. Chira, Proc. Natl. Acad. Sci. 76, 111 (1979).

35. L. Mets and R. J. Gulotty, unpublished results.

36. R. F. P. Gregory, Biochemistry of Photosynthesis (Wiley, London, 1977). 2nd ed.

37. D. H. Waldeck, A. J. Cross, D. B. McDonald and G. R. Fleming, J. Chem. Phys. 74, 3381 (1981).

38. D. H. Waldeck and G. R. Fleming, J. Phys. Chem. 85, 2614 (1981).

39. A. J. Cross, D. H. Waldeck and G. R. Fleming, J. Chem. Phys. in press (1983). 
40. G. L. Eesley, M. D. Levenson and W. M. Tolles, IEEE, J. Quant. Electron QE14, 45 (1978).

41. A. Owyoung, IEEE, J. Quant. Electron QE14, 192 (1978).

42. N. J. Frigo, H. Mahr and D. J. Erskine, IEEE, J. Quant. Electron QE18, 192 (1982).

43. D. H. Waldeck, W. T. Lotshaw, D. B. McDonald and G. R. Fleming, Chem. Phys. Lett. 88, 297 (1982).

44. J. J. Song, J. H. Lee and M. D. Levenson, Phys. Rev. A17, 1439 (1978).

45. T. Yajima, H. Souma and Y. Ishida, Phys. Rev. A17, 324 (1978).

46. H. Souma, E. Heilwell and R. M. Hochstrasser, J. Chem. Phys. 76, 5693 (1982). 